\title{
Fatores de risco para transmissão da Hanseníase
}

\author{
Risk factors for Leprosy transmission \\ Factores de riesgo para la transmisión de la Lepra
}

\section{Andréia Soprani dos Santos', Denise Silveira de Castro", Aloísio FalQueto" \\ 'Escola Superior de Ciências da Santa Casa de Misericórdia. Vitória, ES \\ I'Universidade Federal do Espírito Santo. Programa de Pós-Graduação em Saúde Coletiva. Vitória, ES}

Submissão: $25 / 08 / 2008$

Aprovação: 10/10/2008

\section{RESUMO}

Estudo caso controle Que objetivou identificar fatores individuais de risco relacionados à transmissão da doença. O grupo caso, composto por 90 pacientes de hanseníase notificados no SINAN entre 2003 e 2006; o grupo controle, constituído por 270 indivíduos sadios, pareados por sexo e faixa etária. Houve associação significativa entre a ocorrência da doença e a presença atual $(O R=2,9)$ e antiga $(O R=5,0)$ de hanseníase entre parentes co-sangüíneos. Sabendo-se que o exame único dos contatos, no ato do diagnóstico, detecta uma parcela mínima dos casos, propõe-se a realização de exames periódicos dos contatos de hansenianos a fim de detectar os novos casos.

Descritores: Hanseníase; Epidemiologia; Doenças endêmicas; Fatores de risco.

\begin{abstract}
This case-control study aimed to identify individual risk factors regarding the transmission of leprosy. The group case represented by 90 cases of leprosy sick people pontificated at SINAN during 2003 and 2006 and a group control constituted by 270 healthy people, paired by gender and age. There was significant statistical between occurrence of the disease and its current presence $(\mathrm{OR}: 2,9)$ and old cases $(\mathrm{OR}=$ $5,0)$ of leprosy among co-sanguine relatives. Knowing that the only exam of the contact in the act of the diagnostic detects a minimum part of the new cases, it is proposed to execute periodical examinations of the contact of leprosy for detecting the new cases.
\end{abstract}

Descriptors: Leprosy; Epidemiology; Endemic diseases; Risk factors.

\section{RESUMEN}

Estudio caso controle Que objetivó identificar factores individuales del riesgo relacionados a transmisión de la enfermedad. El grupo caso, compuesto por 90 pacientes con lepra notificados en el SINAN entre 2003 y 2006; y el grupo controle, constituido por 270 individuos saludables, pareados por sexo y edad. Hubo asociación significativa entre la ocurrencia de la enfermedad y la presencia actual $(\mathrm{OR}=2,9)$ y antigua $(\mathrm{OR}=5,0)$ de la lepra entre parientes co-sanguíneos. Sabiéndose que el examen único de los contactos en el acto del diagnóstico detecta una parcela mínima de los casos, se propone a la realización del examen periódico de los contactos de leprosos a fin de detectar los nuevos casos.

Descriptores: Lepra; Epidemiología; Enfermedades endémicas; Factores de riesgo. 


\section{INTRODUÇÃO}

Desde a Antigüidade, a hanseníase tem sido considerada uma doença contagiosa, mutilante e incurável, provocando uma atitude preconceituosa de rejeição e discriminação ao seu portador, normalmente um excluído da sociedade. As referências mais remotas datam de 600 a.C. e procedem da Ásia Que, juntamente com a África, pode ser considerada o berço da doença $a^{(I)}$.

A doença é transmitida principalmente por meio do convívio com os doentes do tipo virchowiano ou dimorfo que ainda não foram diagnosticados e não iniciaram tratamento. Esses indivíduos possuem carga bacilar suficiente para favorecer a transmissão. As principais fontes de bactérias são provavelmente as mucosas das vias aéreas superiores. $\mathrm{O}$ bacilo de Hansen tem a capacidade de infectar grande número de indivíduos, no entanto, poucos adoecem. Essa propriedade não é função apenas das características intrínsecas da bactéria, mas depende, sobretudo, de sua relação com o hospedeiro e do grau de endemicidade do meio ${ }^{(1,2)}$.

A infecção é considerada de fácil diagnóstico e terapêutica. $\mathrm{O}$ esquema de Polieuimioterapia (PQT), recomendado para o tratamento dos doentes, leva à cura em períodos de tempo relativamente curtos, sendo possível desenvolver atividades de controle da doença mesmo em municípios minimamente estruturados. No entanto, a situação da hanseníase em âmbito mundial e nacional ainda é preocupante e se observa Que muitos países ainda não conseguiram eliminar essa doença.

A partir do ano de 1991, o Brasil assume o compromisso de eliminar a hanseníase até 2000, Quando se objetivava alcançar o índice de menos de I doente a cada 10.000 habitantes, alvo preconizado pela organização Mundial de Saúde (OMS).

Visto Que a meta prevista para o ano de 2000 e anos seguintes não foi atingida, o objetivo agora é postergado para o período de 2006 a 2010 , a fim de Que os municípios busquem individualmente atingir um patamar de controle. O eixo central do plano é utilizar a rede de atenção básica junto às unidades de saúde da família. Os secretários municipais de saúde devem desempenhar papel estratégico ao assumir a responsabilidade pela eliminação da doença em seus municípios ${ }^{(3)}$.

Esse processo de reestruturação do modelo assistencial trouxe novas perspectivas, das Quais vale destacar a mudança do paradigma assistencial, vindo de um modelo assistencialista voltado para as especialidades médicas, agora centrado na vigilância em saúde focada no sujeito e na família. É depositada nas equipes de saúde da família a atuação diante dos diversos contextos pertencentes ao foco familiar.

O estado do Espírito Santo é considerado, de acordo com o grau de endemicidade das diferentes unidades federativas e macrorregiões, área de alta prevalência, atingindo em 2005 uma prevalência de 4,54/10.000 hab e detecção de 4,44/10.000 hab. Especificamente, o município de laguaré, localizado na macrorregião Norte do estado, foi caracterizado como área hiperendêmica $(\geq 20$ casos/10.000 hab.). Atualmente, este município apresenta um coeficiente de prevalência de 15,43/10.000 hab, para o ano de $2006^{(4)}$.

Na tentativa de controlar a doença, o município de Jaguaré incentiva a política de descentralização para atenção básica e a implantação de ações de controle da hanseníase por meio do Programa de Saúde da Família (PSF). O programa de controle da hanseníase é uma das prioridades no município, por ser uma área endêmica, tendo sido desenvolvida uma estratégia de ação rigorosa, com treinamentos e capacitações de todos os profissionais de saúde. As ações preventivas, promocionais e curativas foram intensificadas na tentativa de melhorar o perfil epidemiológico do município e vêm sendo realizadas com sucesso pelas equipes, o que evidencia um forte comprometimento de todos os profissionais com a busca por melhorias na perspectiva clínica e epidemiológica do paciente e, por conseguinte, do município.

Nesse sentido, o trabalho tem como objetivo identificar fatores individuais de risco relacionados à transmissão da hanseníase em um município endêmico no interior do estado do Espírito Santo. Essa necessidade é explicada pelo número considerável de pacientes com hanseníase no Brasil, em vários estados da nação e, particularmente, no município de laguaré, por ser essa infecção uma Questão de saúde pública e fazer parte do planejamento estabelecido pelo Ministério da Saúde, Qual seja, a eliminação da doença até o ano de 2010 e pela dificuldade enfrentada pelo serviço de saúde em baixar os índices até o nível proposto no período estabelecido pela OMS.

\section{METODOLOGIA}

Trata-se de um estudo de caso-controle. O grupo caso foi composto por pacientes diagnosticados como casos novos de hanseníase e notificados no banco de dados oficial brasileiro Sistema de Informação Nacional de Agravos de Notificações (SINAN) entre os anos de 2003 e 2006 na rede de saúde do município de laguaré - ES, até completar o Quantitativo de 90 entrevistados. Foram excluídos do estudo os casos Que entraram no sistema de notificação como recidivas, reingressos, casos transferidos de outros municípios ou estados, ou casos de hanseníase Que não residiam no município de Jaguaré - ES.

O grupo controle foi composto por indivíduos moradores vizinhos do caso Que não apresentam diagnóstico de hanseníase, possuem o mesmo sexo (masculino ou feminino) do paciente caso e a idade compreendida em uma faixa etária com variação máxima de 05 anos. A entrevista era antecedida à realização de exame dermatoneurológico. Foram excluídos do estudo os controles que, após o exame dermatoneurológico, apresentavam-se como suspeitos a caso novo de hanseníase.

A escolha do grupo controle ou de comparação obedeceu ao princípio de máxima similaridade com os casos, exceto pelo critério de presença da doença ou agravo.

A medida de associação entre a doença e o fator de risco utilizado foi a razão de chances odds ratio (OR). O cálculo amostral levou em consideração os resultados de um estudo semelhante onde a variável (fator) utilizado foi a escolaridade, com um odds $=2,05$, percentual de expostos de $45 \%$ entre os controles e um poder de $80 \%$, bem como alfa $=0,05$.

O tamanho da amostra necessária, com precisão, intervalo de confiança, poder do teste de $80 \%$ e alfa $=0,05$, foi calculado para um número de casos igual a 90 e número de controles igual a 270 , isto devido a escolha de proporção de três controles por caso. A amostra total foi constituída de 360 entrevistados.

Para a coleta de dados, foi realizado um ineuérito domiciliar por meio da entrevista semi-estruturada contendo variáveis de 
identificação do entrevistado, sócio-econômicas e demográficas, hábitos de vida e relacionadas com a hanseníase.

Para a realização do trabalho de campo por meio das visitas domiciliares aos 90 casos e 270 controles, partiu-se de um grupo composto pelo investigador responsável pelo estudo e com auxílio dos enfermeiros integrantes do PSF, bem como a participação ativa dos agentes comunitários de saúde de cada território. O inquérito domiciliar foi realizado nos meses de novembro de 2005 a setembro de 2006.

Para análise dos resultados foram utilizados pacotes computacionais: Excel 2003, EPI-INFO, SPSS. Após essa análise o estudo foi submetido a um modelo de análise dito regressão logística. O modelo seguiu ajuste por meio do software SPSS 13.0, através do método Backward (passa atrás), baseado no Teste de Wald.

$\mathrm{O}$ trabalho foi submetido à avaliação e julgamento, tendo sido aprovado pelo comitê de ética em pesQuisa do Hospital Universitário Cassiano Antônio de Moraes (HUCAM). O município no qual foi realizada a pesQuisa recebeu orientação prévia sobre a investigação, tendo concordado em contribuir para o desenvolvimento do trabalho. Todos os pacientes foram informados a respeito da pesquisa e de seu objetivo. Quando cientes, deram autorização prévia, assinando o termo de consentimento livre e esclarecido.

\section{RESULTADOS E DISCUSSÃO}

Foram entrevistadas 360 pessoas, com média de idade de 42,8 anos, tendo como idade mínima 6 anos e idade máxima 84 anos. $\mathrm{O}$ tamanho médio da residência ficou em torno de $52,8 \mathrm{~m}^{2}$. O estudo também identificou casas com a metragem de $9 \mathrm{~m}^{2}$ e casas com até $250 \mathrm{~m}^{2}$. Em relação ao consumo de álcool, foi encontrado valor médio igual a $48,3 \mathrm{ml} / \mathrm{semana}$, um valor mínimo de $3 \mathrm{ml} / \mathrm{semana}$ e o máximo de $280 \mathrm{ml} / \mathrm{semana}$. Para o consumo de cigarro, nota-se a média de consumo de 13,7 cigarros/dia, Quantidade mínima de I cigarro/dia e valor máximo de 60 cigarros/dia.

Em relação ao tipo de ocupação mais encontrada entre casos e controles, houve predomínio de domésticas 93 (25,8\%), seguido de lavradores 79 (21,9\%), aposentados 51 (14,2\%) e braçais I 5 (4,2\%). Quanto ao nível de escolaridade entre os casos, foi observado que a maioria 55,6\% possui nível fundamental, e 24,4\% são analfabetos. Na classificação das raças, o estudo identificou no grupo caso discreto predomínio da raça parda 38,9\% seguido de $37,8 \%$ da raça negra. No que diz respeito à renda salarial, o estudo mostrou predomínio da faixa de um a três salários mínimos no grupo caso $68,9 \%$, seguido de 24,4\% com renda menor Que I salário mínimo.

$\mathrm{Na}$ entrevista, ao serem Questionados a respeito do diagnóstico da hanseníase, a maioria dos casos $69 \%$ referiu ter procurado o posto de saúde devido ao aparecimento de manchas pelo corpo, 25,5\% descobriram em um dia de consulta na unidade de saúde e 5,5\% foram encaminhados ao posto de saúde pelo agente comunitário.

No grupo caso, assim como no grupo controle, foi observado Que $75,6 \%$ usam água tratada, 16,9\% usam água de poço e 7,5\% usam água de nascente. Com relação ao consumo, 54,2\% bebem água tratada e 45,8\% bebem água não tratada.

A variável tipo de residência mostrou predomínio, entre os casos, do tipo tijolo 77,8\% e do tipo madeira 12,2\%. Em relação à Quantidade de moradores nas casas, o grupo caso apresentou uma média de 4,3 pessoas e o grupo controle 4,0 pessoas. Quanto à procedência do grupo caso, observou-se Que o maior número $76,6 \%$ possui como local de origem e morada o município de Jaguaré. Os demais casos 23,3\% são advindos de outras cidades.

Com relação a casos atuais de hanseníase entre parentes consangüíneos, $86,4 \%$ não possuem casos na família e $13.6 \%$ possuem casos na família. Para essa pergunta p-valor 0,012 e OR 2,374, essa variável encontrou significância estatística. Quanto a ter casos antigos de hanseníase entre parentes consangüíneos, 83 , I \% não possui casos na família e $16,9 \%$ possui casos na família. Para essa Questão p-valor 0,000 e OR 4,203, assim, essa variável também encontrou significância estatística.

O estudo revelou, após o emprego da regressão logística, Que ter casos atuais de hanseníase na família está associado a um risco 2,9 vezes maior de um membro sadio dessa família contrair a doença; e ter casos antigos de hanseníase na família está associado à possibilidade 5,0 vezes maior de um membro sadio dessa família contrair a doença.

Aceita-se que a transmissão da hanseníase acontece de pessoa a pessoa. O risco de desenvolvimento da doença é cerca de 5 a 10 vezes mais alto se um membro da família já manifestou a doença. Para os contatos intra-domiciliares, o risco de desenvolver a hanseníase é maior para aQueles Que convivem com o doente antes de ele iniciar o tratamento ${ }^{(5)}$.

Outros pesquisadores, ao entrevistar 20 famílias de pacientes com hanseníase na região de DuQue de Caxias no Rio de Janeiro, observaram Que há predominância (69/75) da consangüinidade entre os Que adoeceram e Que a possibilidade de adoecer entre os consangüíneos foi 2,8 vezes maior do Que entre os não consangüíneos $^{(6)}$.

Ainda em 1998, a Organização Mundial de Saúde já enfatizava o papel relevante do contato intra-domiciliar na epidemiologia da doença e, em especial, tem chamado a atenção para possibilidades estratégicas para o controle e a eliminação da doença, o que tem sido reiterado como um objetivo básico.

Embora a idade seja um fator utilizado nesse estudo para pareamento dos grupos, não se pode deixar de destacar dois fatos importantes: a presença da doença entre crianças, com idade mínima de 6 anos e a média de idade encontrada no grupo caso de 43 anos. Este destaca a ocorrência da doença em idade tardia, o Que reflete um período de incubação longo, isso pode significar Que um único exame dos contatos no momento do diagnóstico do doente não detecta a maioria dos futuros casos. E aquele nos mostra que a hanseníase continua sendo transmitida intensamente dentro da comunidade e sugere contágio nos primeiros anos de vida, o Que remete ao fato da transmissão intra-domiciliar e familiar.

$\mathrm{Na}$ classificação das raças, o estudo identificou no grupo caso discreto predomínio da raça parda $38,9 \%$ seguido de $37,8 \%$ da raça negra. Demais autores, encontraram em seus estudos realizados no Nordeste a raça parda como predominante ${ }^{(7,8)}$.

No Que diz respeito à renda salarial, o estudo mostrou predomínio da faixa de um a três salários mínimos no grupo caso de $68,9 \%$, seguido de $24,4 \%$ com renda menor Que I salário mínimo. Esses dados são semelhantes ao percentual encontrado por outros pesquisadores, Que sugerem Que os enfermos de hanseníase pertencem à classe social média baixa ${ }^{(9-11)}$.

Os tipos de ocupação mais encontrados entre os entrevistados foram os de doméstica 25,8\%, seguido de lavrador $21,9 \%$. Em estudo 
Tabela 1. Distribuição das variáveis Quantitativas entre os 360 entrevistados, compondo os grupos de casos (90) e controles (270), no município de Jaguaré - ES no período de nov de 2005 a set de 2006.

\begin{tabular}{cccccc}
\hline Variáveis ouantitativas & $\mathrm{n}$ & Mínimo & Máximo & Média & DP \\
\hline Idade & 360 & 6 & 84 & 42,8 & 17,7 \\
Tamanho da casa $\left(\mathrm{m}^{2}\right)$ & 360 & 9,0 & 250,0 & 52,8 & 32,64 \\
Número de moradores na residência & 360 & 1 & 11 & 4,1 & 1,8 \\
$*$ Quantidade de álcool ingerida (ml/semana) & 142 & 3 & 280 & 48,3 & 57,6 \\
$* *$ Quantidade de cigarros (cigarros/dia) & 112 & 1 & 60 & 13,7 & 12,6 \\
\hline
\end{tabular}

*Só foram considerados os indivíduos Que fazem uso de bebida alcoólica

** Só foram considerados os indivíduos que fazem uso de cigarro

Tabela 2. Distribuição das variáveis Qualitativas entre os grupos caso (90) Hanseníase e controle (270) pesQuisados no município de Jaguaré - ES, no período de nov 2005 a set 2006.

\begin{tabular}{|c|c|c|c|c|c|c|c|c|c|}
\hline \multirow[t]{2}{*}{ Variáveis eualitativas } & & & \multicolumn{2}{|c|}{ Grupo } & \multirow{2}{*}{ Total } & \multirow{2}{*}{ p-valor } & \multirow{2}{*}{ OR } & \multicolumn{2}{|c|}{ IC 95\% para OR } \\
\hline & & & Hanseníase & Controle & & & & linf & Lsup \\
\hline \multirow{4}{*}{ Sexo } & \multirow{2}{*}{ Feminino } & $n$ & 46 & 138 & 184 & \multirow{4}{*}{1,000} & & & \\
\hline & & $\%$ & 51,1 & 51,1 & 51,1 & & & & \\
\hline & \multirow{2}{*}{ Masculino } & $\mathrm{n}$ & 44 & 132 & 176 & & & & \\
\hline & & $\%$ & 48,9 & 48,9 & 48,9 & & & & \\
\hline \multirow{6}{*}{ Raça } & \multirow{2}{*}{ Negro } & $n$ & 34 & 101 & 135 & \multirow{6}{*}{0,388} & & & \\
\hline & & $\%$ & 37,8 & 37,4 & 37,5 & & & & \\
\hline & & $n$ & 35 & 122 & 157 & & \multirow{2}{*}{0,852} & \multirow{2}{*}{0,496} & \multirow{2}{*}{1,463} \\
\hline & Pardo & $\%$ & 38,9 & 45,2 & 43,6 & & & & \\
\hline & \multirow{2}{*}{ Branco } & $\mathrm{n}$ & 21 & 47 & 68 & & \multirow{2}{*}{1,327} & \multirow{2}{*}{0,697} & \multirow{2}{*}{2,529} \\
\hline & & $\%$ & 23,3 & 17,4 & 18,9 & & & & \\
\hline \multirow{6}{*}{ Escolaridade } & \multirow{2}{*}{$\begin{array}{c}\text { Nível } \\
\text { fundamental }\end{array}$} & $n$ & 50 & 154 & 204 & \multirow{6}{*}{0,388} & \multirow{2}{*}{1,092} & \multirow{2}{*}{0,616} & \multirow{2}{*}{1,937} \\
\hline & & $\%$ & 55,6 & 57,2 & 56,8 & & & & \\
\hline & \multirow{2}{*}{ Nível médio } & $n$ & 16 & 38 & 53 & & \multirow{2}{*}{1,455} & \multirow{2}{*}{0,684} & \multirow{2}{*}{3,096} \\
\hline & & $\%$ & 17,8 & 13,8 & 14,8 & & & & \\
\hline & \multirow{2}{*}{ Nível superior } & $\mathrm{n}$ & 2 & 4 & 6 & & \multirow{2}{*}{1,682} & 0.289 & 9804 \\
\hline & & $\%$ & 2,2 & 1,5 & 1,7 & & & & , 004 \\
\hline & Menor oue 1 & $\bar{n}$ & 22 & 51 & 73 & & & & \\
\hline & IVIenor Que I & $\%$ & 24,4 & 18,9 & 20,3 & & & & \\
\hline & & $\mathrm{n}$ & 62 & 206 & 268 & 0332 & 0698 & 0202 & 240 \\
\hline Kenaa & Entre I e 3 & $\%$ & 68,9 & 76,3 & 74,4 & 0,333 & 0,698 & 0,393 & 1,240 \\
\hline & Maior oue 3 & $n$ & 6 & 13 & 19 & & 070 & 0.360 & 3.179 \\
\hline & ivalor vues & $\%$ & 6,7 & 4,8 & 5,3 & & 1,070 & 0,500 & $3,17)$ \\
\hline
\end{tabular}

realizado por outro autor, com pacientes de hanseníase, foi observado o predomínio de lavrador, doméstica e estudante ${ }^{(11)}$.

Em relação ao nível de escolaridade entre os casos, foi observado Que a maioria $(55,6 \%)$ possui nível fundamental e $(24,4 \%)$ são analfabetos. Parra ${ }^{(1)}$, em seu estudo, encontrou um valor de $75 \%$ para o paciente Que tinha algum grau de instrução seja ele básico ou médio, e um valor de 15\% para analfabeto. Esse dado é também semelhante ao descrito por Pedroso ${ }^{(12)}$.

Em estudo caso-controle realizado por Kerr-Pontes ${ }^{(13)}$, com pacientes de hanseníase, a variável baixa escolaridade mostrou-se como um fator de risco, obtendo resultado de OR: I,81. É sabido Que o nível educacional de uma nação demonstra o seu estrato populacional. Pode-se considerar também a dificuldade no acesso aos serviços de saúde e na promoção da saúde e prevenção de doenças.

Na entrevista, ao serem Questionados a respeito do diagnóstico da hanseníase, a maioria dos casos $69 \%$ referiu ter procurado o posto de saúde devido ao aparecimento de manchas pelo corpo. Esse fato pode demonstrar o conhecimento dessa população em relação aos sinais da doença, identificando um bom trabalho das equipes de PSF no processo de educação em saúde.

O Ministério da Saúde recomenda a participação das equipes para aumentar o conhecimento da população a respeito dos estágios iniciais da doença, uma estratégia para colaborar no diagnóstico precoce da hanseníase.

Para Cunha ${ }^{(14)}$, em estudo feito em um município endêmico do 
Tabela 3. Distribuição dos casos atuais e antigos entre parentes consangüíneos nos grupos caso (90) Hanseníase e controle (270) peseuisados no município de Jaguaré - ES, no período de nov 2005 a set 2006.

\begin{tabular}{|c|c|c|c|c|c|c|c|c|c|}
\hline \multirow[t]{2}{*}{ Variáveis Qualitativas } & & & \multicolumn{2}{|c|}{ Grupo } & \multirow{2}{*}{ Total } & \multirow{2}{*}{ p-valor } & \multirow{2}{*}{ OR } & \multicolumn{2}{|c|}{ IC 95\% para OR } \\
\hline & & & Hanseníase & Controle & & & & linf & Isup \\
\hline \multirow{4}{*}{$\begin{array}{l}\text { Casos atuais de } \\
\text { hanseníase entre } \\
\text { parentes co- } \\
\text { sanguíneos }\end{array}$} & \multirow{2}{*}{ Sim } & $n$ & 20 & 29 & 49 & \multirow{4}{*}{0,012} & \multirow{4}{*}{2,374} & \multirow{4}{*}{1,266} & \multirow{4}{*}{4,453} \\
\hline & & $\%$ & 22,2 & 10,7 & 13,6 & & & & \\
\hline & \multirow{2}{*}{ Não } & $\mathrm{n}$ & 70 & 241 & 311 & & & & \\
\hline & & $\%$ & 77,8 & 89,3 & 86,4 & & & & \\
\hline \multirow{4}{*}{$\begin{array}{l}\text { Casos antigos de } \\
\text { hanseníase entre } \\
\text { parentes co- } \\
\text { sanguíneos }\end{array}$} & \multirow{2}{*}{ Sim } & $\mathrm{n}$ & 31 & 30 & 61 & \multirow{4}{*}{0,000} & \multirow{4}{*}{4,203} & \multirow{4}{*}{2,360} & \multirow{4}{*}{7,486} \\
\hline & & $\%$ & 34,4 & 11,1 & 16,9 & & & & \\
\hline & \multirow{2}{*}{ Não } & $\mathrm{n}$ & 59 & 240 & 299 & & & & \\
\hline & & $\%$ & 65,6 & 88,9 & 83,1 & & & & \\
\hline
\end{tabular}

Tabela 4. Estatística das variáveis casos atuais e casos antigos submetidas à Regressão logística.

\begin{tabular}{|c|c|c|c|c|c|c|c|c|}
\hline & \multirow{2}{*}{ Variáveis no Modelo } & \multirow{2}{*}{ B } & \multirow{2}{*}{ Erro Padrão (B) } & \multirow{2}{*}{ Wald } & \multirow{2}{*}{ p-valor } & \multirow{2}{*}{$\operatorname{Exp}(B)$} & \multicolumn{2}{|c|}{ I.C. de $95 \%$ para OR } \\
\hline & & & & & & & $\operatorname{linf}$ & Lsup \\
\hline \multirow{3}{*}{ Modelo 9} & Casos atuais & 1,097 & 0,358 & 9,41 & 0,002 & 2,996 & 1,486 & 6,041 \\
\hline & Casos antigos & 1,614 & 0,323 & 25,02 & 0,000 & 5,024 & 2,669 & 9,456 \\
\hline & Constante & $-1,670$ & 0,177 & 88,86 & 0,000 & 0,188 & & \\
\hline
\end{tabular}

Rio de Janeiro, a realização de atividades educativas junto à comunidade a partir das unidades de saúde proporcionou à população melhoria de conhecimento sobre a doença e esse fato pode influenciar no aumento da detecção de casos novos na forma inicial da doença.

Em relação ao tipo de casa e o tamanho da residência, resultado semelhante foi observado por 0 rtiz $^{(15)}$. 0 s autores observaram que $73,1 \%$ dos entrevistados habitam em casas de tijolo e $26,9 \%$ habitam em casas de madeira. Em estudo realizado por Andrade ${ }^{(16)}$, no município do Rio de Janeiro, a respeito de fatores associados ao domicílio e à família na determinação da hanseníase, foi observado que em $45,9 \%$ dos domicílios de doentes de hanseníase residem mais de cinco pessoas e $57,6 \%$ das casas têm $50 \mathrm{~m}^{2}$ ou menos. 0 fato de apresentar casas com dimensões muito pequenas e um bom quantitativo de moradores poderia facilitar o processo de transmissão da doença e ser apontado como fator de risco.

Quanto à procedência do grupo caso, alguns autores encontraram relação semelhante, como O liveira ${ }^{(17)}$ e Aquino ${ }^{(8)}$. Em seu estudo, Oliveira ${ }^{(17)}$ referiu que $9,6 \%$ dos pacientes atendidos foram provenientes de outras cidades. Esses autores atribuíram o fato à estigmatização da doença e ao medo do paciente em ser descoberto pelos demais. Isso os levaria a procurar atendimento em outras cidades.

Para Andrade ${ }^{(16)}$, considerando o território de estudo, a imigração não parece ter contribuído de modo relevante mediante a introdução de novas fontes de infecção. Ao contrário, como a endemia já estava instalada na área central mais antiga, os imigrantes estariam contribuindo para manter o processo de transmissão por meio do aporte de susceptíveis.

Pacientes diagnosticados com hanseníase hoje talvez tenham transmitido a doença para outras pessoas na família ou na comunidade por um longo período em que a doença ainda não havia sido diagnosticada. Nesse sentido, o exame dos contatos intradomiciliares torna-se fundamental para a descoberta de novos casos e orientação adequada, alertando quanto aos sinais característicos da doença.

Em estudo realizado por A quino ${ }^{(8)}$, para avaliar um programa de controle de hanseníase, foi observado que um número reduzido de contatos vem sendo contemplado com tal medida. D os contatos dos pacientes paucibacilares, nenhum foi examinado, e dos multibacilares, apenas $16,8 \%$. M agalhães $\&$ Rojas ${ }^{(18)}$, constataram que em todo o Brasil o número de contatos examinados é menor que $30 \%$, de todos os comunicantes registrados.

\section{CONCLUSÃO}

Diante de várias questões levantadas acerca da endemia hansênica, o presente estudo vem contribuir para reforçar a afirmativa da transmissão da doença em âmbito familiar. No decorrer da pesquisa, - estudo revelo u que ter casos atuais de hanseníase na família aumenta em 2,9 vezes o risco de contrair a doença. E para os casos antigos de doença na família, o risco aumenta para possibilidade 5,0 vezes maior de desenvolver a patologia.

Ao estimar que o diagnóstico dos casos seja feito, em média, aos 42 anos de idade, deduz-se que a doença geralmente se manifesta vários anos após o contato com o doente. Levando-se em consideração o longo período de incubação da doença e ainda o fator de risco ter na família um paciente com a hanseníase, sugere-se novas avaliações dos contatos intra-domiciliares, mesmo anos depois do membro da família ter encerrado o tratamento. Dessa maneira, seria possível avaliar de fato se aquele familiar exposto desenvolveu a doença a partir do caso familiar contaminado. 
Ao considerar que nesse estudo $69 \%$ dos casos foram diagnosticados a partir de sua ida à unidade de saúde do PSF, a atuação da atenção primária em saúde junto ao $\mathrm{PNCH}$ torna-se essencial, pois é por meio dela que o usuário do SUS procura por um atendimento. Nesse sentido, um bom atendimento e qualidade no serviço prestado ao usuário e às famílias é a garantia para detecção e diagnóstico de casos novos de hanseníase, com orientação adequada sobre as características e tratamento da doença.

A dificuldade em alcançar a meta de controle da hanseníase pode estar relacionada também à própria particularidade da endemia local. A existência de conglomerados populacionais pode sustentar os índices de transmissão em níveis elevados, assim como os hábitos

\section{REFERÊNCIAS}

1. Fundação Nacional de Saúde (BR). Guia de vigilância

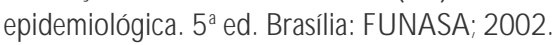

2. Marteli CMT, Stefani MMA, Penna GO, Andrade ALSS. Endemias e epidemias brasileiras, desafios e perspectives de investigação científica: hanseníase. Rev Bras Epidemiol 2002; 5(3): 273-85.

3. Ministério da Saúde (BR). Informações de saúde. Brasília: M inistério da Saúde; 2007.

4. Prefeitura M unicipal de Jaguaré (ES). Secretaria M unicipal de Saúde. Plano municipal de eliminação da hanseníase. Jaguaré: Prefeitura M unicipal; 2005.

5. Organização Panamericana de Saúde, Organização Mundial de Saúde. Hanseníase hoje. Boletim de Eliminação da Hanseníase das Américas 1999.

6. Oliveira MLWDR, Duraes SMB, Guedes LS, Cunha MD, Cavaliere FAM. Estudos de 20 focos familiares de hanseníase no município de Duque de Caxias, Rio de Janeiro. An Bras D ermatol 2005; 80(3): 295-300.

7. Fonseca PHM . Hanseníase no Estado do M aranhão: análise de 5.274 casos. Arq Bras Med 1983; 57: 175-7.

8. Aquino DMC, Santos JS, Costa JM L. A valiação do programa de controle da hanseníase em um município hiperendêmico do Estado do Maranhão, Brasil, 1991-1995. Cad Saúde Pública 2003; 19(1): 119-25.

9. Serruya J. Hanseníase no município do Rio de Janeiro. Anais Bras Dermatol 1981; 56: 251-4.

10. Aquino DMC, Caldas AJM, Silva AAM, Costa JML. Perfil do pacientes com hanseníase em área hiperendêmica da A mazônia do de vida estabelecidos em cada território, as condições sanitárias e econômicas capazes de contribuir para persistência do agravo. N esse sentido, o pequeno tamanho das habitações evidencia as condições sócio-econômicas em que vivem os doentes e as pessoas propensas a contrair a doença.

Finalmente, o exame dos contatos domiciliares dos casos novos diagnosticados, aliado à informação efetiva da população sobre a doença, o rastreamento de novos casos e a educação permanente dos profissionais de saúde tornam-se estratégias principais para 0 diagnóstico e tratamento da hanseníase e poderão facilitar o processo de eliminação do agravo proposto para até 0 ano de 2010.

Maranhão, Brasil. Rev Soc Bras M ed Trop 2003; 36(1): 57-64.

11. Parra MC. Caracterización sócio-econômica de los leprosos atendidos en la unidade de dermatología sanitaria de M aracaibo, Venezuela: un estudio de casos. Cad Saúde Pública 1996; 12(2): 225-31.

12. Pedroso M. Incapacidades físicas em hanseníase: estudo multicêntrico da realidade brasileira. Anais Bras D ermatol 1989; 64: 301-6.

13. Kerr-Pontes LRS, Barreto M L, Evangelista CM N, Rodrigues LC, Heukelbach J, Feldmeier H. Socioeconomic, environment, and behavioural risk factors for leprosy in $\mathrm{N}$ orth-east Brazil: results of a case-control study. Int J Epidemiol 2006; 27: 1-7.

14. Cunha M D, Cavaliere FAM, Hércules FM, D uraes SMB, O liveira MLWDR. Os indicadores da hanseníase e as estratégias de eliminação da doença em município endêmico do estado do Rio de Janeiro, Brasil. Cad Saúde Pública 2007; 23(5): 1187 97.

15. Ortiz LC, Hoz FL, Léon Cl, Guerrero MI, Gamboa LA, Araújo MJ. Caracterización clínica y sociodemográfica de casos nuevos de lepra en municipios endémicos y no endémicos de Colombia. Rev Salud Pública 2004; 6(1): 50-63.

16. Andrade VLG, Sabroza PC, Araújo AJG. Fatores associados ao domicílio e à família na determinação da Hanseníase, Rio de Janeiro, Brasil. Cad Saúde Pública 1994; 10(2): 281-92.

17. O liveira M HP. Incapacidades físicas em hanseníase [tese]. São Paulo (SP): Universidade de São Paulo; 1987.

18. Magalhães M CC, Rojas LI. Evolución de la endemia de la lepra en Brasil. Rev Bras Epidemiol 2005; 8(4): 342-55. 